\title{
La implantación en España de la formación profesional dual: perspectivas
}

\author{
Oriol Homs \\ Centro de Iniciativas e Investigaciones Europeas en el Mediterráneo (CIDEM) \\ oriol.homs@gmail.com
}

Resumen: El objetivo de este artículo es exponer, por un lado, que internacionalmente cada vez se acepta más la visión de la existencia de una gran variedad de modelos en los que se alterna la formación escolar y el aprendizaje en el puesto de trabajo, de origen muy diverso, que no puede reducirse a la hipótesis de una progresiva implantación de un modelo germánico que se extiende mundialmente; y por otro, que la formación dual aún se halla en un estadio muy incipiente en España.

Palabras clave: formación profesional; formación profesional dual; formación en centros de trabajo.

The implementation in Spain of the Dual Vocational Education and Training: perspectives

Abstract: The objective of this article is to expose, on the one hand, that at the international level it is increasingly accepted the vision of the existence of a variety of models in which school education and workplace learning alternate, of very diverse source, which can not be reduced to the bypothesis of a progressive introduction of a Germanic model that extends worldwide. On the other hand, the «dual training» is still in a very nascent stage in Spain.

Keywords: Vocational Education and Training; Dual Vocational Education; Training, Workplace Training. 


\section{Antecedentes y enfoque inicial}

La implantación en España de la llamada formación profesional dual no está exenta de ciertas contradicciones. Normalmente se conoce por formación profesional dual al modelo alemán de formación profesional basado en la alternancia entre una formación escolar en un centro formativo y otra en un lugar de trabajo, organizado por una empresa. Este modelo, con sus variantes, se ha extendido a otros países centroeuropeos, principalmente Austria, Suiza y Luxemburgo. Desde una perspectiva más técnica y general, estos modelos de formación profesional se caracterizan porque se basan en la figura del aprendiz, por lo que en los ámbitos europeo e internacional se conocen por sus señas en inglés como apprenticeship vocational training models. Así pues, programas formativos basados en la figura del aprendiz existen prácticamente en todos los países, con más o menos grado de desarrollo e importancia en sus respectivos sistemas nacionales de formación profesional.

Lo curioso es que en el momento en el que se implantan en España programas de este tipo se utiliza el concepto de formación dual, aunque el modelo que se aplica no tiene las características del modelo alemán ni centroeuropeo. Ya fuera por la presión alemana para que se implantara este tipo de programas en España, ya que en su momento no estaba en la agenda de la política educativa española, o bien porque el modelo alemán goza en España de un reconocimiento de calidad y prestigio, se optó por llamarlo formación dual. En cambio, no se utilizó el concepto de aprendiz, que había tenido su recorrido en la tradición laboral española, seguramente porque era más controvertido, ya que la opinión general estaba dividida entre los que defendían que era un buen sistema de inserción de los jóvenes en el mercado de trabajo y los que lo criticaban por suponer que era un sistema de explotación laboral de los jóvenes.

Sin embargo, tampoco se ha utilizado el concepto de formación en alternancia, que estaba teniendo una progresiva incorporación en el sistema español de formación profesional, y bajo cuya denominación, la Administración española, en sus informes para la Comisión Europea, situaba los programas de formación ocupacional que compaginan formación y empleo, como las conocidas escuelas taller, casas de oficio y otras fórmulas en las que el aprendizaje se forma simultaneando, bajo diferentes formas: la formación de tipo escolar y la formación en el trabajo. El concepto de formación en alternancia es el que han utilizado otros países que han incorporado a sus sistemas formativos la combinación de formación escolar y formación en el trabajo como método de aprendizaje profesional, como es el caso, principalmente, de Francia. 
La realidad es que, desde una perspectiva comparativa, internacionalmente cada vez se acepta más la visión de la existencia de una gran variedad de modelos en los que se alterna la formación escolar y el aprendizaje en el puesto de trabajo, de origen muy diverso, que no puede reducirse a la hipótesis de una progresiva implantación de un modelo germánico que se extiende mundialmente. Véanse los resultados del informe del Centro Europeo para el Desarrollo de la Formación Profesional (CEDEFOP) sobre Governance and financing apprenticeship (CEDEFOP, 2016), o bien los trabajos del International Network on Innovative Apprenticeship (INAP) sobre esta materia (Deitmer y otros, 2013).

Es una lástima que no se hayan enlazado tradición e innovación para desarrollar en España un método de formación de gran interés, sea cual sea la fórmula que se utilice, y que tiene un gran porvenir para regular la transición de los jóvenes a la vida activa. No obstante, aún se está a tiempo, puesto que la formación dual todavía se halla en un estadio muy incipiente en España.

Se decía que la implantación de la formación dual en España no seguía los cánones de la formación dual alemana, y en los próximos apartados se entrará en detalle en esta afirmación, pero antes se resaltará la importancia de los aspectos culturales que implica para el contexto español la incorporación de este tipo de formación.

\section{La formación dualः un cambio cultural}

El sistema español de formación profesional está clasificado internacionalmente en el área de los modelos escolares. Esta es la tradición secular del sistema español, en el que la formación profesional se imparte en centros formativos específicos dotados de instalaciones y talleres adecuados para el aprendizaje práctico de oficios y profesiones. Este modelo conlleva un amplio conjunto de valores, símbolos y conceptos muy anclados en la cultura española de la formación profesional.

Algunos de estos elementos culturales chocan abiertamente con algunos de los valores que caracterizan los sistemas basados en el aprendizaje en la empresa. Por ello, se considera que deberían tenerse en cuenta los aspectos culturales que implica la implantación en España de un modelo formativo que se basa en presupuestos culturales alternativos.

Las diferencias culturales más significativas entre ambos modelos se refieren a los siguientes aspectos:

- La preponderancia de la formación teórica sobre la práctica. Es comúnmente aceptado que para formar a un buen profesional es necesaria una sólida formación teórica e instrumental sobre la que construir el desarrollo de las habilidades prácticas y específicas de cada profesión. No obstante, la 
discrepancia entre ambos modelos radica en la relación que se establece entre estos. En los modelos escolares prima la formación teórica construida académicamente sobre la formación práctica, que tiene una posición subordinada porque se piensa que ya se desarrollará en la actividad profesional. En cambio, los modelos basados en la formación en el puesto de trabajo se estructuran a través de la capacidad de aprendizaje de las actividades prácticas, es decir, siguen las pautas de los postulados del training by doing. Para ellos, la realización práctica de una actividad profesional es la que permite estructurar los contenidos de las competencias cognitivas, las habilidades y las aptitudes necesarias para formar a un buen profesional. La teoría se concibe como un complemento necesario para asentar bien los aprendizajes prácticos y para fortalecer la capacidad de innovación que proporciona el conocer los porqués de la práctica laboral. Ambas posiciones no se niegan mutuamente, sino que son diferentes por el carácter estructurante del contenido de los aprendizajes profesionales: para uno es el conocimiento teórico y para el otro es el conocimiento práctico.

- Se aprende en la escuela y se práctica en la empresa. Para los modelos escolares, la formación en la empresa se concibe como una práctica de lo que se ha aprendido en el centro de formación y, a lo sumo, como el aprendizaje de las competencias transversales del saber ser y del saber estar. En cambio, para los otros modelos, la formación en la empresa no se concibe como una práctica, sino como una fuente de aprendizaje, no solo de las competencias transversales, sino también de las competencias técnicas y específicas de la profesión. Se parte del concepto de que ciertas competencias es mejor que se aprendan en un contexto escolar y otras, en un contexto laboral. El modelo español contiene un elemento de suma singularidad, que es la formación en el centro de trabajo (FCT), en la que el alumno realiza una estancia de entre 350 y 700 horas en la empresa al finalizar su formación escolar en un ciclo de formación profesional. Pocos países tienen esta fórmula para todos los alumnos de los programas profesionales. Sin embargo, la FCT se considera como unas prácticas en un contexto laboral que complementan la formación en el centro de formación. Esta concepción es distinta de la que se le otorga a la estancia en la empresa en los sistemas basados en la figura del aprendiz o en los sistemas de alternancia.

- El rol de la empresa. En los sistemas escolares, la empresa es un colaborador receptor de estudiantes para enriquecer su formación. Esta colaboración le facilita a la empresa la identificación de buenos estudiantes que 
puedan ser de su interés para tenerlos en cuenta cuando sea necesario contratar a futuros profesionales. En cambio, especialmente en el modelo germánico, la empresa es el pilar del sistema. La empresa asume la función formativa de los futuros profesionales del país, no solamente de sus propios futuros profesionales, puesto que forma a jóvenes entre los que escogerá a los mejores para su plantilla cuando lo requiera. Por otra parte, esta función formativa le permite a la empresa fortalecer sus procesos de producción al ejercer una función tutorial, y además, poder disponer en la etapa final del proceso de aprendizaje de una mano de obra que complemente su plantilla. En los modelos escolares, la función de la empresa es más pasiva y no llega a asumir directamente la responsabilidad formativa, sino solo la responsabilidad de acoger y mostrar cómo se trabaja.

- La evaluación de los aprendizajes es competencia del profesor del centro formativo. Como consecuencia de los aspectos anteriores, en los modelos escolares, es evidentemente que el profesor del centro formativo es el que tiene la responsabilidad y la competencia para evaluar los aprendizajes realizados por los alumnos, incluso los adquiridos durante su estancia en la empresa, aunque en este caso, con la colaboración del tutor empresarial. En cambio, en los otros modelos, cada uno evalúa los aprendizajes de los que se ha responsabilizado. La evaluación final es compartida. En el caso de Alemania, los tribunales que otorgan las valoraciones finales son tripartitos, están organizados por las cámaras de comercio en los que participan los agentes sociales y por los tutores de la empresa y del centro de formación.

- La formación como una cuestión de relaciones laborales. El corolario de los aspectos anteriores es que, en los otros modelos, la formación profesional está incardinada en las relaciones laborales y es una corresponsabilidad entre el Estado y la empresa. La regulación suele ser estatal, pero el actor clave es la empresa, y por ende, los agentes que participan en la empresa: empresarios y sindicatos. Por ello, hay tantos sistemas de formación en alternancia como sistemas laborales. Según las tradiciones y los modelos de relaciones laborales se estructura el rol del alumno-trabajador y el papel de los sindicatos. En el modelo germánico, el alumno es un trabajador sometido a una regulación laboral específica, en la que el sindicato juega un papel importante en el ámbito tanto de la empresa como general.

Como puede observarse, las diferencias son de calado. Una vez introducido en España el modelo dual, seguramente el principal obstáculo para su arraigo sean los aspectos culturales, tanto entre los reguladores y gestores como entre los 
principales actores: las empresas, los centros de formación y los alumnos y sus familias. Los procesos de cambio cultural siempre son lentos, por lo que, seguramente, el desarrollo de este nuevo paradigma formativo llevará su tiempo.

Por eso, tendría que haberse buscado un hilo conductor que enlazara con la tradición cultural de la formación profesional española para que se fueran introduciendo los nuevos conceptos progresivamente. Seguramente, el punto de mejor anclaje de la formación en alternancia podría ser la FCT y la reconversión de los programas de formación y empleo en el ámbito de la formación ocupacional.

La FCT está ya muy consolidada y ha sido validada por su aportación a la mejora de la formación profesional y a la inserción de los jóvenes en el mercado de trabajo. Hoy ya es la principal vía de acceso al empleo de los titulados de formación profesional. Sin embargo, la calidad de estas prácticas aún es muy variable, depende de la empresa colaboradora y del planteamiento del centro formativo.

Una iniciativa para mejorar la calidad de la FCT, que alargara el periodo de estancia en la empresa; regulara mejor el carácter laboral de las prácticas, a semejanza de la regulación sobre los convenios de prácticas no laborales; avanzara el momento de las prácticas hacia el inicio de los ciclos formativos; diera más importancia al papel de los tutores de empresa; y ampliara la responsabilidad formativa de la empresa, seguramente, abriría una vía de mejora de la formación profesional actual y, al mismo tiempo, iría introduciendo los nuevos conceptos culturales de la formación en alternancia.

Una propuesta de estas características promovería un campo de innovación y de experimentación en el que participarían los centros más interesados en él, que arrastraría con el tiempo al conjunto del sistema de formación hacia una concepción más orientada hacia las necesidades de cualificación de las empresas y de la incorporación de los jóvenes al mercado de trabajo.

También hay que sospesar hacia dónde quiere orientarse el sistema de formación profesional español: ¿hacia un modelo que tienda como tal a configurarse como un modelo en alternancia, o bien hacia un modelo mayoritariamente escolar, pero que abre una vía minoritaria complementaria basada en la alternancia? Es decir, ¿se quiere ir hacia un modelo de alternancia o hacia un programa de alternancia que conviva con un programa general de tipo escolar?

En los países germánicos impera la primera orientación, aunque con enormes dificultades, ya que tampoco consiguen que el modelo dual sea ampliamente mayoritario, puesto que también convive con otros modelos más escolares con un peso muy elevado. Sin embargo, culturalmente dominan las concepciones de los modelos de alternancia. En estos países, la forma de entender la formación profesional es la propia de la formación dual. 
En cambio, en los países que no tienen influencia germánica, la tónica predominante es la segunda opción, en la que los programas de alternancia son minoritarios y conviven con el modelo preponderante típicamente escolar, con muchas variantes en la combinación entre formación y trabajo.

De todos modos, en el caso español, aunque parece que se ha optado por la segunda opción, se ha tomado una buena decisión al integrar los proyectos de formación dual en el marco de la regulación general de la formación profesional, con una visión unitaria e integrada. Es decir, no se ha optado por crear un programa concreto con una certificación o titulación específicas para estos programas, como se hizo en su día, de forma errónea, con las escuelas taller o con las casas de oficio. La formación dual es una modalidad diferente de cursar unos mismos programas con una misma titulación. Esta decisión facilitará el desarrollo de los nuevos modelos de alternancia. No es este el caso de muchos países que han optado por introducir programas de alternancia, pero desagregados del resto del sistema mayoritario, con lo cual han hipotecado su crecimiento o han complicado la relación integrada del conjunto del sistema de formación.

El acierto de la visión unitaria en España ha dado ya sus frutos, más rápidamente de lo que se esperaba inicialmente, seguramente debido también a un efecto cultural. La formación dual en España goza de un prestigio que atrae a los jóvenes, las empresas y los centros de formación.

En efecto, los primeros ensayos de proyectos de formación dual han sido protagonizados por los centros más innovadores del país, que se han interesado por ofrecerles a sus alumnos y a las empresas a las que están vinculados a través de la FCT la nueva modalidad. El prestigio de la formación alemana y que los mejores centros con los mejores alumnos hayan optado por introducir proyectos duales está favoreciendo su desarrollo, a pesar de las dificultades en su gestión inicial. La formación dual se ha asociado con una formación de calidad y con mayores perspectivas de inserción laboral para sus alumnos, cuestión importante en una época de elevadas tasas de paro juvenil.

\section{La regulación de la formación dual}

La introducción formal de la formación dual en España no se produjo hasta la publicación del Real Decreto 1529, de 8 de noviembre de 2012, a pesar de que existía, como ya se ha mencionado, una larga experiencia en programas de alternancia entre formación y empleo, aunque de reducidas dimensiones y circunscritos a la formación para el empleo, especialmente con los programas de escuelas taller y casas de oficios. 
El Real Decreto 1529/2012 abre un periodo experimental en el que se regulan bajo el concepto de formación dual un amplio conjunto de posibilidades para articular la formación escolar con la práctica laboral en las empresas. Estas posibilidades abarcan desde la formación en un centro escolar con prácticas en las empresas hasta la formación totalmente impartida en una empresa, pasando por situaciones intermedias de mayor o menor implicación empresarial.

De hecho, la implantación de los programas de formación dual en España se ha dejado en manos de la iniciativa política de las comunidades autónomas. Hasta el momento, las regiones que han demostrado más interés son el País Vasco, Cataluña, la Comunidad Valenciana, Castilla la Mancha y Andalucía. Aunque hay también iniciativas en otras comunidades, suelen tener un alcance escaso hasta el momento. En el curso 2014 había en España unos 10000 alumnos en régimen de formación dual, lo que representa aproximadamente un 1,4 \% del total de alumnos matriculados en los ciclos de formación profesional. En este mismo curso había implicados unos 375 centros de formación y unas 1570 empresas. Cada comunidad ha seguido diferentes pautas, por lo que hoy existe un amplio abanico de experiencias en marcha, cuyo principal resultado ha sido promover la flexibilización del modelo escolar vigente hasta el momento.

El Real Decreto 1529/2012 tiene como objetivo principal desarrollar reglamentariamente la legislación sobre el contrato laboral de formación y aprendizaje para agilizar y facilitar su utilización, introduciendo la regulación de determinados aspectos de la formación dual. Hay que aclarar que este decreto tiene como motivación principal la regulación laboral de un contrato pensado para favorecer la inserción laboral de los jóvenes en el mercado de trabajo, y que el legislador lo aprovecha para introducir criterios a fin de promover la implantación en España de la formación dual. Es importante aclarar esta cuestión porque el decreto no aborda directamente, ni fundamentalmente, la regulación de un modelo completo de formación dual, sino que básicamente tiene una orientación hacia la inserción laboral de los jóvenes. Esta dependencia inicial hacia el contrato de formación y aprendizaje condicionará la implantación de la formación dual, ya que el contrato de formación y aprendizaje no está pensado principalmente para esto, sino para favorecer la inserción laboral de los jóvenes. A pesar de ello, el decreto ha abierto en España el fomento de los programas de formación dual y ha provocado también cambios de interés en la oferta formativa de formación profesional.

La formación dual se define como las acciones e iniciativas formativas mixtas de empleo y formación en régimen de alternancia entre la actividad laboral en una empresa y las actividades formativas recibidas en el marco de los dos subsistemas de formación profesionalः el educativo y el de la formación para el empleo. 
Esta definición es importante porque marca toda la filosofía del decreto: incluye todo el sistema, tanto la formación profesional inicial como la formación para el empleo, y lo particulariza en acciones e iniciativas. Es decir, no pretende definir un modelo, sino que propone un marco regulador para que todas las acciones e iniciativas formativas que pretendan la alternancia entre formación y empleo, y que cumplan con los requisitos del decreto, puedan ser consideradas como una formación dual. De hecho, abre una vía de experimentación, lo que permite que las partes tomen la iniciativa del desarrollo de proyectos duales. De esta forma, incluso, considera incluibles dentro de la formación dual las acciones de alternancia que no utilicen el contrato de formación y aprendizaje.

El decreto señala dos actores principales en el desarrollo de los proyectos de formación dual: la empresa y el centro de formación, y un actor gestor directo: los servicios de empleo estatales y autonómicos. Es decir, si una empresa desea ofrecerle un contrato de formación y aprendizaje a un joven, debe concertar un convenio con un centro de formación para definir la actividad formativa, y todo el proceso debe ser supervisado y aceptado por los servicios estatales y/o autonómicos de empleo. De forma indirecta entran en escena las comunidades autónomas como responsables de los centros de formación y de la gestión de los sistemas autonómicos de formación profesional y de los titulares de los servicios de empleo autonómicos. De hecho, la iniciativa concreta está en manos de las empresas y los centros de formación, pero su desarrollo dependerá, en gran medida, del interés y la implicación de las comunidades autónomas. Aunque esta regulación del decreto es de ámbito estatal y puede aplicarla cualquier empresa, se puede constatar que en las comunidades en las que sus gobiernos no se han implicado en el desarrollo de los proyectos duales, estos no se han desarrollado significativamente.

Los agentes sociales no aparecen en todo el decreto, solamente han sido consultados en los órganos de gobernanza del sistema de formación profesional. Su única implicación es que las empresas deben comunicarle a la representación de los trabajadores en la empresa los acuerdos suscritos con los centros de formación y con los jóvenes para la formación dual, en los que deberán constar las personas contratadas para la formación, el puesto de trabajo que deberán desempeñar y el contenido de la formación que deberán realizar. Ellos mismos han criticado abiertamente el no haber tenido en consideración a los agentes sociales en la regulación de los proyectos duales, que, de hecho, es una de las causas de su poca actividad en este ámbito hasta el momento. Aunque esta posición está cambiando hacia una mayor implicación, puesto que se está reclamando la participación directa de los agentes sociales no solo en la gobernanza, sino también en su implementación en los ámbitos tanto regional como de empresa y sectorial. 
En la práctica, el desarrollo de los programas de formación dual ha tenido como base el interés y la implicación de los gobiernos regionales. En estas comunidades, han sido las empresas de mayor tamaño y las empresas pequeñas y medianas más innovadoras las que se han interesado por los proyectos duales. No obstante, también, muy a menudo, la iniciativa ha surgido por parte de los centros formativos, tanto públicos como privados, más innovadores. Es decir, se ha configurado una colaboración de excelencia entre empresas, centros de formación y comunidades autónomas basada en la innovación que ha prestigiado las experiencias y los proyectos de formación dual. Esto ha configurado un mapa diverso de la implementación de la formación dual, con variaciones según la comunidad autónoma.

El País Vasco es la comunidad autónoma que tiene el sistema de formación profesional más desarrollado en España, con una larga tradición de colaboración entre centros de formación y empresas. Antes del Real Decreto 1529/2012, esta comunidad ya había puesto en marcha proyectos de alternancia, como el Ikasi eta Lan y, posteriormente, el Hezibi. El Gobierno vasco aprobó el 2 de junio de 2015 el Decreto 83/2015, en el que se regula la formación profesional dual en régimen de alternancia. Las autoridades vascas se decantaron por un modelo basado en los centros de formación con la participación de las empresas, ya sea con contratos de formación y aprendizaje o con becas formativas. La iniciativa la tienen los centros de formación. Los alumnos que participan en los programas de alternancia llevan a cabo un primer curso en el centro y, una vez aprobado, durante el segundo curso, alternan su formación entre el centro y la empresa que asume ciertas actividades formativas. De hecho, este modelo es una extensión de los cursos estándares, en el que se alarga el periodo de prácticas en la empresa y hay una mayor implicación de la empresa en la formación del alumno. La evaluación y certificación de los aprendizajes continúa estando bajo la responsabilidad del centro de formación. El centro formativo tiene autonomía para flexibilizar y adaptar el contenido formativo a las necesidades de la empresa, bajo el control del Gobierno autonómico. Los agentes sociales tampoco participan en su implementación, tan solo se les informa a través de la representación de los trabajadores en la empresa de las condiciones de los convenios suscritos con los centros formativos.

En el caso de Andalucía, el Departamento de Educación de la Junta de Andalucía ha optado por abrir una convocatoria de proyectos de formación dual dirigidos a centros públicos y privados de formación profesional. Para el curso 20162017 se han autorizado 234 proyectos en todo el territorio de la comunidad.

El interés del caso andaluz radica en la adaptación a las características del tejido productivo de la comunidad. Los proyectos liderados por los centros de 
formación implican a varias empresas y a las autoridades locales en su desarrollo. Cabe destacar en este caso que el alumno puede acceder a la actividad laboral después del primer trimestre de formación en el centro siguiendo una modalidad rotativa de las actividades formativas entre varias empresas. De este modo, el alumno puede realizar su actividad formativa en varias empresas. Esto le permite un aprendizaje más variado y completo dado el escaso tamaño de la gran mayoría de las empresas en Andalucía. La actividad laboral en la empresa puede realizarse de forma alternativa dividiendo el tiempo semanal entre el centro y la empresa o de forma consecutiva, con periodos totalmente escolarizados y periodos totalmente laborales en la empresa.

Otras comunidades autónomas, como la Comunidad Valenciana o Castilla la Mancha, presentan elementos de ambos modelos. En el caso de la Comunidad Valenciana cabe destacar la implicación de las cámaras de comercio y de las asociaciones empresariales en la promoción de la formación dual y en el asesoramiento al Gobierno regional. A pesar de los intentos por dotar de una mayor participación a las cámaras en la formación dual en España, no se ha logrado aún el suficiente consenso sobre su papel en el ámbito estatal.

En Cataluña existe un variado abanico de experiencias de centros formativos que están realizando proyectos de formación dual. Por su grado de consolidación e innovación, merece destacar el caso del IES Comte de Rius de Tarragona, que ha desarrollado la formación dual en la formación tanto inicial como para el empleo en colaboración con las empresas de su entorno, principalmente del sector petroquímico, una iniciativa en la que también se han implicado los sindicatos de la zona.

La diferente situación en cada comunidad autónoma refleja una amplia dispersión de proyectos y procedimientos, que en este periodo de experimentación ha tenido la virtud de facilitar su implementación. Otro efecto positivo de una regulación experimental abierta que cabe señalar es que ha facilitado un proceso de flexibilización de la oferta de formación profesional para adaptarse mejor a las necesidades de las empresas. En muchos casos se han establecido convenios entre empresas o gremios para flexibilizar los currículos de los ciclos a fin de incorporar algunos aprendizajes propios de una empresa o un sector, lo que ha permitido que se adapten a las necesidades empresariales. Esta posibilidad ya existía en la legislación sobre formación inicial, pero se utilizaba poco. En cambio, en los últimos años se ha utilizado más, especialmente en Cataluña.

Por otra parte, tanto la flexibilización curricular como los proyectos de formación dual han fortalecido los lazos entre centros de formación y empresas, lo que ha redundado en una colaboración más estrecha y en una mejor aproximación 
a las necesidades de las empresas, que era uno de los puntos débiles del sistema escolar de formación profesional en España.

La contrapartida es una cierta confusión sobre qué se entiende por formación dual, ya que en la realidad abarca un amplio abanico de proyectos, cuyo punto en común es que alternan formación y trabajo, pero que en el procedimiento, el estatus del estudiante, la responsabilidad empresarial y la relación entre la empresa y el centro difieren considerablemente.

Ir más allá de la etapa de experimentación y apostar por la consolidación de un sistema de alternancia en España requeriría la superación de los límites de la situación actual. Por ahora, los beneficios son superiores a los costes, pero también es cierto que en esta etapa inicial han sido los centros más innovadores y las empresas, en general, más innovadoras y competitivas las que han apostado por colaborar y asumir conjuntamente el proceso formativo, y es probable que ampliar la zona de influencia al conjunto del tejido productivo y al mapa de centros formativos sea más lento y costoso que lo que se ha conseguido en poco tiempo.

Esto requeriría abordar algunas cuestiones, que con una evaluación en profundidad de las experiencias en curso podrían ponerse de relieve:

- El papel de los agentes sociales. Uno de los objetivos que debe conseguirse es una mayor implicación de las empresas en la formación de los profesionales del futuro, y por tanto, su implicación en el desarrollo de programas de alternancia es vital para su generalización. Al hablar de una empresa tan importante es la dirección empresarial para que apueste por este tipo de formación y movilice los recursos humanos, técnicos y económicos para ello como lo son el propio personal y sus representantes sindicales, ya que asumir la formación de nuevos profesionales implica a toda la plantilla, ya sea a algunos como tutores directos y a otros como compañeros de trabajo. Es toda la empresa, todo su equipo humano, quien debe asumir esta responsabilidad. Si en una empresa hay un ambiente escéptico respecto a la modalidad de alternancia porque se teme que frene la ampliación de la plantilla, o bien porque exige esfuerzos de reorganización o tareas suplementarias, difícilmente la dirección de la empresa facilitará su implicación en los nuevos programas. En este sentido, son ya visibles cambios de actitud entre los agentes sociales, del lado tanto sindical como empresarial, mantenidos al margen durante la etapa experimental. Hoy, tanto los sindicatos como las organizaciones empresariales se inclinan más a tener un mayor protagonismo en la organización y el seguimiento de los proyectos de formación en alternancia. Son significativos los debates, por ahora internos, en el seno de la Confederación Española de 
Organizaciones Empresariales (CEOE) al respecto. Las propuestas que se están debatiendo proponen que las empresas y las organizaciones empresariales tengan un mayor protagonismo en la gestión de los fondos provenientes de las cotizaciones a la seguridad social en concepto de formación. En el caso de la formación dual, proponen que el 50 \% lo financien fondos públicos y privados, con las aportaciones de las empresas a la seguridad social y los gastos de las empresas dedicados a la contratación de aprendices.

- La regulación del estatuto de los estudiantes-trabajadores. Ya se ha mencionado que el contrato de formación y aprendizaje no se adapta bien a las necesidades de la regulación prelaboral de los jóvenes durante su estancia en la empresa en el marco de una formación en alternancia. El modelo de los convenios de prácticas no laborales podría servir de ejemplo para una regulación más acorde, que debería consensuarse en el ámbito del diálogo social entre los agentes sociales y la Administración pública.

- La adaptación de la formación a las necesidades de las pymes. El tejido empresarial español es el que es, y por tanto, pensar que una pyme con menos de cinco trabajadores, como es el caso de la inmensa mayoría de las empresas españolas, puede asumir responsabilidades formativas es ilusorio si quiere construirse un sistema de calidad profesionalizante. La experiencia andaluza de implicar a los municipios y buscar formas de mutualización con varias pymes del esfuerzo formativo puede también constituir una referencia interesante para la reflexión. Seguramente en estos casos, la unidad empresa no sea la más adecuada y haya que buscar unidades más amplias, ya sea en el seno de un clúster, de un grupo empresarial, de las cámaras, o bien de una zona determinada a través del municipio.

- El papel de los tutores de empresa. Si la empresa debe asumir funciones más amplias de tipo formativo, debe prepararse el terreno para que sea factible. El papel de los tutores y las condiciones de su trabajo para ejercer esta función serán relevantes. Esto afectará a la negociación colectiva en la empresa o el sector, a la regulación de su formación y a su reconocimiento en el proceso evaluativo de los aprendizajes realizados.

Abordar todas estas cuestiones seguramente requiere reformular el Real Decreto 1529/2012 para abrir una nueva etapa con un nuevo impulso para la extensión de la formación en alternancia. Esto implicaría también agilizar los procesos de actualización del Catálogo Nacional de Cualificaciones, gestionado por el Instituto Nacional de las Cualificaciones (INCUAL), para facilitar la adaptación de 
la formación a las necesidades cambiantes de las empresas ante el rápido proceso de innovación productiva, tecnológica y organizativa.

Es paradójico que grupos empresariales como Volkswagen, en España, regulen sus programas de formación dual siguiendo las directrices del grupo central alemán, y añadan un tercer año de formación dual para complementar el currículo español con nuevos requerimientos competenciales a fin de adaptarse a las necesidades productivas de la fabricación de los nuevos productos.

El caso español presenta otra singularidad, ya que no solo se ha iniciado la introducción de la formación en alternancia en la formación inicial, sino que también se ha incorporado a los certificados de profesionalidad, quizá en menor extensión, debido, principalmente, a la rigidez de los contenidos formativos de los certificados, que no permiten un grado de flexibilidad como en la formación inicial, cuando debería suceder lo contrario, y seguramente también a la fuerte burocratización de su gestión a través de los servicios públicos de empleo. Ambos aspectos deberían revisarse para conseguir una mayor extensión de la formación en alternancia en esta importante área del sistema de formación.

\section{Referencias}

Centro Europeo para el Desarrollo de la Formación Profesional (CEDEFOP) (2016): Governance and financing apprenticeship, Salónica: CEDEFOP.

Deitmer y otros (eds.) (2013): The architecture of innovative apprenticeship, Nueva York y Londres: Springer. 